\title{
The Role of Hypofractionated Radiotherapy in Prostate Cancer
}

\author{
Linus C. Benjamin ${ }^{1}$ • Alison C. Tree ${ }^{1,2}$ - David P. Dearnaley ${ }^{1,2}$
}

Published online: 25 March 2017

(C) The Author(s) 2017. This article is published with open access at Springerlink.com

\begin{abstract}
Purpose of Review It is now accepted that prostate cancer has a low alpha/beta ratio, establishing a strong basis for hypofractionation of prostate radiotherapy. This review focuses on the rationale for hypofractionation and presents the evidence base for establishing moderate hypofractionation for localised disease as the new standard of care. The emerging evidence for extreme hypofractionation in managing localized and oligometastatic prostate cancer is reviewed.

Recent Findings The 5-year efficacy and toxicity outcomes from four phase III studies have been published within the last 12 months. These studies randomizing over 6000 patients to conventional fractionation (1.8-2.0 Gy per fraction) or moderate hypofractionation (3.0-3.4 Gy per fraction). They demonstrate hypofractionation to be non-inferior to conventional fractionation.

Summary Moderate hypofractionation for localized prostate cancer is safe and effective. There is a growing body of evidence in support of extreme hypofractionation for localized prostate cancer. Extreme hypofractionation may have a role in
\end{abstract}

This article is part of the Topical Collection on Genitourinary Cancers

David P. Dearnaley

David.Dearnaley@icr.ac.uk

Linus C. Benjamin

linus.benjamin@icr.ac.uk

Alison C. Tree

Alison.tree@rmh.nhs.uk

1 Division of Radiotherapy and Imaging, The Institute of Cancer Research and The Royal Marsden Hospital NHS Foundation Trust, Downs Road, Sutton Surrey, SM2 5PT London, UK

2 Royal Marsden NHS Foundation Trust and the Institute of Cancer Research, Downs Road, Sutton Surrey, SM2 5PT London, UK managing prostate oligometastases, but further studies are needed.

Keywords Prostate cancer $\cdot$ Radiotherapy ·

Hypofractionation · CHHiP · HYPRO

\section{Introduction}

Prostate cancer ( $\mathrm{PCa})$ is the second most commonly diagnosed male malignancy [1] in the Western world, with the majority of patients having organ-confined disease at presentation [2].

Radical or curative radiotherapy has been traditionally given with a conventionally fractionated schedule, using daily dose of 1.8-2.0 Gy over 7-8 weeks, to a total dose of 74$79.2 \mathrm{~Gy}$.

The dose of 1.8-2.0 Gy per fraction in conventional fractionation $(\mathrm{CF})$ is based on the presumed relative sensitivity of malignant and normal tissue. The radiobiology and response of tumours and normal tissue to total dose and dose per fraction has been the subject of intense research for more than 30 years [3, 4]. These studies led to the development of the linear-quadratic model, which describes the relationship between cell survival, dose and dose per fraction [5]. In the linear-quadratic model, the response of tissue to fraction size is described by the alpha/beta ratio $(\alpha / \beta)$. The $\alpha / \beta$ for most tumours is $>8 \mathrm{~Gy}$, while that for late-responding normal tissue is estimated at 3-4 Gy. For these tumours, CF at 1.8-2.0 Gy per fraction results in an improvement in the therapeutic ratio.

In parallel advances in physics, engineering, computing and imaging have been channelled into the development of image-guided intensity-modulated radiotherapy. The improvements in imaging give better target definition, and it is now possible to accurately deliver highly conformal 
treatment. This has made it possible to both reduce radiationrelated side-effects and escalate dose. Several phase III studies in prostate cancer have shown that increasing dose, improves biochemical disease-free survival, with acceptable acute and long-term toxicities [6-9]. If CF is used, dose-escalated radiotherapy is now the standard of care.

Dose escalation has been achieved through and increase in the number of fractions delivered. This has resulted in prolongation of total treatment time, with an increased number of hospital visits for patients, as well as greater departmental workload and higher costs. With an improvement in the understanding of the radiobiology of $\mathrm{PCa}$, hypofractionation has become an attractive means of dose escalation, without prolonging treatment duration.

\section{Rationale for Hypofractionation}

The theoretical basis for hypofractionation in $\mathrm{PCa}$ is the comparatively low $\alpha / \beta$ for PCa. Evidence supporting the hypothesis for a very low $\alpha / \beta$ for PCa has become available in the last two decades, including pre-clinical and clinical data. In 1999, a study of 367 patients calculated an $\alpha / \beta$ of 1.5 Gy (95\% CI 0.8-2.2) [10]. A subsequent study [11] with 1020 patients treated with external beam radiotherapy and brachytherapy derived the same value $(1.5 ; 95 \%$ CI $1.25-1.75)$. These early findings have been supported in a retrospective analysis of 6000 patients treated with external beam radiotherapy [12], which calculated an $\alpha / \beta$ of 1.4 (95\% CI 0.9-2.2), with no significant difference in the calculated ratio between different risk groups or with androgen deprivation. In conjunction with other studies [13-15], the $\alpha / \beta$ of PCa can be estimated at 1.4-1.9 Gy. This estimate does not take into account the potential effects of treatment duration or accelerated repopulation [14].

The low $\alpha / \beta$ estimates for PCa suggest a greater sensitivity to increasing fraction size, raising the possibility of doseescalation through hypofractionation. The $\alpha / \beta$ for doselimiting organs in prostate radiotherapy is postulated to be comparatively higher (rectum and bladder; $\alpha / \beta 3-5$ Gy). This forms the theoretical basis for an improvement in the therapeutic ratio of radiotherapy with larger fraction sizes, while delivering an isoeffective dose to the prostate.

\section{Moderate Hypofractionation}

Moderate hypofractionation (MH) refers to the delivery of 2.4-4.0 Gy per fraction, daily, over 4-6 weeks.

Two systematic reviews [16, 17] of the randomized evidence for prostate MH had previously concluded that there was insufficient evidence to demonstrate that $\mathrm{MH}$ produces improved outcome compared to CF. However, new data from four randomized studies published within the last 12 months $[18 \bullet \bullet, 19-21,22 \bullet, 23 \bullet, 24 \bullet]$ are now available. Collectively, 6357 patients have been randomized to $\mathrm{CF}$ (1.8-2.0 Gy per fraction) or $\mathrm{MH}$ (3.0 3.4 Gy per fraction), and 5-year efficacy outcomes and toxicity profiles have been reported. These results show that $\mathrm{MH}$ is as well tolerated and as clinically effective as $\mathrm{CF}$, with the obvious economic and practical advantages associated with shorter treatment durations.

\section{Moderate Hypofractionation for Localized Disease}

Randomized data comparing $\mathrm{MH}$ to $\mathrm{CF}$ includes two early trials, four modern superiority trials, and three modern noninferiority trials (Table 1).

\section{Early Studies}

The earliest randomized studies in $\mathrm{MH}$ were undertaken in Canada [25] and Australia [26] and gave conflicting results. Their design was motivated by logistical benefits of shorter treatment durations, in countries where patients had to travel considerable distances for radiotherapy. The doses in the arms these early studies were not isoeffective, as no assumptions about the $\alpha / \beta$ of PCa were made in the design of the trials.

The Canadian trial [25] enrolled 936 patients with low- or intermediate-risk disease, randomizing them to $66 \mathrm{~Gy} / 33 \mathrm{frac}-$ tions/6.6 weeks or $52.5 \mathrm{~Gy} / 20$ fractions/4 weeks. The biologically effective dose (BED) in the hypofractionated arm was lower than that in the standard arm. The study reported higher rates of 5 -year biochemical failure (60 vs $53 \% ; p<0.05)$ and acute grade $3 / 4$ gastrointestinal (GI) and genitourinary (GU) toxicity (11 vs $7 \%$ ) in the hypofractionated arm.

However, after a median follow-up of 5.7 years, there was no difference in late GI/GU toxicity ( $\geq$ grade 3 late toxicity $3.2 \%$ ) between the two study arms.

The Australian trial [26] randomized 217 patients, with favourable risk $\mathrm{PCa}$, to $64 \mathrm{~Gy} / 32$ fractions/6.4 weeks or $55 \mathrm{~Gy} / 20$ fractions/4 weeks. Treatment was delivered using a 2D, four-field box, technique.

After a median follow-up of 90 months, biochemical disease-free survival was significantly better for the hypofractionated arm (53 vs $34 \% ; p<0.05$ ), with no significant difference in GI/GU toxicity or overall survival. Multivariate analysis revealed $\mathrm{CF}$ to be an independent predictor for biochemical failure and GU toxicity at 4 years.

While these early studies demonstrated the feasibility of $\mathrm{MH}$, their toxicity and efficacy outcomes are not applicable in modern radiotherapy, as the radiotherapy techniques and doses employed in these studies are no longer in keeping with the current standard of care. 


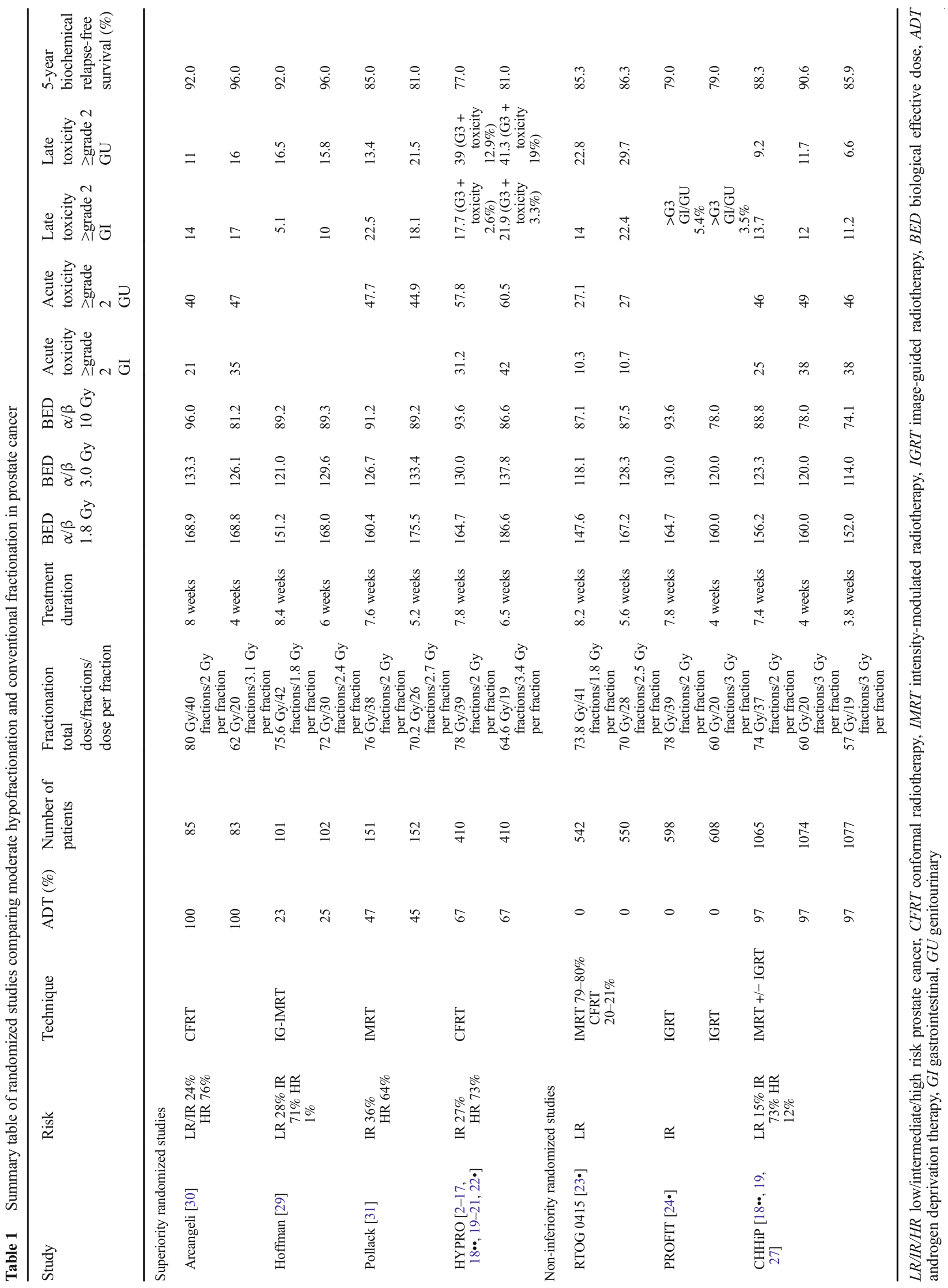




\section{Modern Studies}

The modern MH trials assume that the $\alpha / \beta$ for PCa is $1.5 \mathrm{~Gy}$, and have complementary design, addressing different hypotheses. The superiority studies hypothesize a greater efficacy of hypofractionation with equivalent toxicity, while the noninferiority studies aim to demonstrate equivalent efficacy with reduced or similar toxicity.

There are three large randomized non-inferiority trials (CHHiP [18••, 19, 27], RTOG 0415 [23•] and PROFIT [24-]) evaluating the equivalence of $\mathrm{MH}$ and $\mathrm{CF}$. The doses in the $\mathrm{MH}$ arms in these studies range from 57 to $70 \mathrm{~Gy}$ in 2.5-3.4 Gy per fraction. Overall, these studies demonstrate that the safety and efficacy of MH is similar to that of CF.

The largest non-inferiority randomized study of $\mathrm{MH}$ is the CHHiP study $[18 \bullet \bullet, 19,27]$. This study enrolled 3216 patients from 71 centres in the UK, Ireland, Switzerland and New Zealand. Patients were randomized to $74 \mathrm{~Gy} / 37$ fractions/ 7.4 weeks, 60 Gy/20 fractions/4 weeks, or 57 Gy/19 fractions/3.8 weeks, with treatment delivery using IMRT. The experimental fractionation schedule were designed to be isoeffective for $\alpha / \beta$ of 2.5 Gy (60-Gy schedule) and $1.5 \mathrm{~Gy}$ (57-Gy schedule). Twelve, 73 , and $15 \%$ of patients in this study had low-, intermediate- or high-risk disease, respectively. Short-course hormonal therapy was mandated for patients with intermediate- or high-risk disease.

The primary end point in the CHHiP study was time to biochemical failure, with the critical hazard ratio for noninferiority being 1.208 .

After a median follow-up of 62.4 months, the 5-year biochemical or clinical failure-free survival was found to be $88.3 \%$ in the $74-$ Gy arm (95\% CI 86.0-90.2), $90.6 \%$ in the 60-Gy arm (95\% CI 88.5-92.3), and 85.9\% in the 57-Gy arm (95\% CI 83.4-88.0). The 60-Gy arm was non-inferior to the 74 Gy (HR 0.84, 90\% CI 0.68-1.03; pNI =0.0018). The 57Gy arm was not non-inferior to the 74-Gy arm (HR 1.20, 90\% CI 0.99-1.46). Overall mortality in each arm was similar; 8.6, 6.8 and $8.1 \%$ in the 74, 60 and 57-Gy arm, respectively. There were no statistically significant differences between the arms with respect to distant metastasis rate (3.0, 2.7 and 3.9\% for the 74, 60 and 57Gy arms, respectively).

While acute RTOG GI/GU toxicity had become similar in each arm by 18 weeks, it peaked earlier in the hypofractionated arm (4-5 weeks) compared to the control arm (7-8 weeks). Early GI $\geq$ grade 2 toxicity was significantly higher in the hypofractionated arms; it was $25 \%$ in the $74-G y$ arm, $38 \%$ in the $60-$ Gy arm $(p<0.0001)$ and $38 \%$ in the $57-G y$ arm $(p<0.0001)$.

5 -year clinician and patient-reported side-effects were not significantly different. RTOG grade $\geq 2$ GI toxicity was reported at $13.7,11.9$ and $11.5 \%$ in the 74-, 60- and 57-Gy arms respectively. Grade $\geq 2$ GU toxicity was reported at $9.1,11.7$ and $6.6 \%$ in the 74-, 60- and 57-Gy arms, respectively.
Comparison of the 60- and 57-Gy arms revealed a slightly higher rate of cumulative LENT-SOM grade $\geq 2$ GI toxicity (HR $1.39,95 \%$ CI $1.14-1.70 ; p=0.001$ ) and GU toxicity (HR $1.58,95 \%$ CI $1.13-2.20 ; p=0.007)$.

The CHHiP $[18 \bullet \bullet, 19,27]$ study provides compelling evidence for hypofractionation, with the authors recommending $60 \mathrm{~Gy} / 20$ fractions/4 weeks becoming the new standard of care for the management of localized PCa.

The PROFIT study (NCT00304759) has recently been presented [24•]. The critical hazard ratio for non-inferiority in this study was set at 1.32 . PROFIT [24•] recruited 1206 men with intermediate-risk disease, randomizing them to $60 \mathrm{~Gy} / 20 \mathrm{frac}-$ tions/4 weeks or $78 \mathrm{~Gy} / 39$ fractions/7.8 weeks. All patients in PROFIT [24•] had intermediate-risk disease, with none receiving hormonal therapy.

After a median follow-up of 6 years, no significant difference in 5-year biochemical failure (HR $0.96,90 \%$ CI 0.80 1.15 ), acute $\geq$ grade $3 \mathrm{GI} / \mathrm{GU}$ toxicity, or overall survival have been reported. Interestingly late toxicity was lower in the $\mathrm{MH}$ $\operatorname{arm}(3.5$ vs $5.4 \%$, difference $=-1.9 \%, 95 \% \mathrm{CI}-4.3$ to 0.43$)$.

The authors of PROFIT [24•] conclude that, for patients with intermediate-risk disease, $\mathrm{MH}$ is non-inferior to $\mathrm{CF}$, for both efficacy and acute/late toxicity.

The RTOG 0415 [23•] study randomized 1092 patients, with low-risk disease, to $73.8 \mathrm{~Gy} / 41$ fractions/8.2 weeks or 70 Gy/28 fractions/5.6 weeks. The critical hazard ratios for non-inferiority were set at 1.52 for 5 -year disease-free survival (primary end point), 1.67 for cumulative biochemical recurrence and 1.54 for overall survival.

After a median follow-up of 5.8 years, the $\mathrm{MH}$ arm was reported to be non-inferior to the $\mathrm{CF}$ arm with respect to 5year disease-free survival (HR 0.85, 95\% CI 0.64-1.14; $p<0.001$ ), biochemical recurrence (HR $0.77,95 \%$ CI $0.51-$ $1.17 ; p<0.001$ ) and overall survival (HR $0.95,95 \%$ CI 0.64 $1.41 ; p=0.008$ ).

The acute side effects did not differ significantly in the two arms of the study. The $\mathrm{MH}$ arm had a significantly higher rate of grade $2-3$ late GI toxicity (22.4 vs $14 \%$; RR $1.55-1.59$ ) and grade $2-3$ late GU toxicity ( 29.7 vs $22.8 \%$; RR $1.31-1.59$ ).

The authors concluded that the efficacy of $\mathrm{MH}$ is not inferior to $\mathrm{CF}$, though the late grade 2-3 GI/GU toxicity is higher.

CHHiP [18••, 19, 27], RTOG 0415 [23•] and PROFIT [24•] include patients in different risk groups and differ in the use of hormonal therapy, but all studies give very similar hazard ratios $(<1.0)$ for their primary end points, demonstrating that the efficacy of MH is not inferior to CF. They differ in their late toxicity outcomes.

In contrast to RTOG 0415 [23•],CHHiP [18••, 19, 27] has reported no difference in late toxicity, while PROFIT [24•] reports a lower rate of late toxicity in the hypofractionated arm. These differences may partly be accounted for by the BED in the hypofractionated and control arms of each study. Assuming an $\alpha / \beta$ of $3.0 \mathrm{~Gy}$ for bladder/rectum, the BED in 
the hypofractionated arm is higher than the control arm in RTOG 0415 (128 Gy vs 118 Gy), similar to the control arm in CHHiP [18••, 19, 27] (120 Gy vs 123 Gy), and lower than the control arm in PROFIT [24•] (120 Gy vs 130 Gy).

Comparing PROFIT [24•] and CHHiP [18••, 19, 27], the use of hormonal therapy in some patients in CHHiP appears to improve biochemical control by $10 \%$, although the impact of hypofractionation is similar with or without hormonal therapy.

Finally, the $\alpha / \beta$ estimated by CHHiP $[18 \bullet \bullet, 19,27]$ (1.8 Gy) and PROFIT [24•] (1.3 Gy) are both in keeping with the low range of 1.4-1.9 Gy estimated from meta-analyses and large series [12-15], further re-enforcing the theoretical basis for $\mathrm{MH}$.

In the four modern $\mathrm{MH}$ superiority randomized trials [20, $21,22 \cdot, 28-31]$, the dose in the $\mathrm{MH}$ arms ranges from 62 to 72 Gy in 2.4-3.4 Gy per fraction. Collectively, these studies have not demonstrated any differences in efficacy after 5 years. No differences in metastasis-free, cancer-specific survival or overall survival have been demonstrated.

Hoffman [28, 29] and Pollack [31] compared CF with MH and found no significant difference in 5-year biochemical recurrence-free survival.

Arcangeli [30] recruited 168 patients with high-risk PCa, randomizing them to $80 \mathrm{~Gy} / 40$ fractions/8 weeks or $62 \mathrm{~Gy} / 20$ fractions/4 weeks, in conjunction with 9 months of hormonal therapy.

After a median follow-up of 70 months, a non-significant improvement in actuarial 5-year biochemical recurrence-free survival was demonstrated in the MH arm ( 85 vs $79 \%$; $p=0.065$ ). No significant difference in local or distant recurrence was demonstrated. However, subgroup analysis of patients with a PSA $\leq 20 \mathrm{ng} / \mathrm{ml}$ revealed a significant improvement in 5-year local and distant disease control, in addition to biochemical control.

The HYPRO study $[20,21,22 \cdot]$ is the largest of the MH superiority studies. HYPRO randomized 804 patients, with intermediate- or high-risk disease to $64.6 \mathrm{~Gy} / 19$ fractions/3 fractions per week $/ 6.5$ weeks or $78 \mathrm{~Gy} / 39$ fractions $/ 5$ fractions per week/7.8 weeks. The majority of patients in this study were high-risk ( $>70 \%$ ), with $66 \%$ receiving concomitant hormonal therapy. The primary end point was 5-year relapse-free survival. An additional, non-inferiority, end point was the incidence of $\geq$ grade $2 \mathrm{GI} / \mathrm{GU}$ toxicity, with a critical hazard ratio designated as $1.11 / 1.13$, respectively.

After a median follow-up of 60 months, the 5-year relapsefree survival was not statistically different in the $\mathrm{MH}$ and $\mathrm{CF}$ arms (77.1 vs $80.5 \% ; p=0.36)$.

No differences in acute $\geq$ grade $2 \mathrm{GU}$ toxicity were reported between the $\mathrm{MH}(60.5 \%, 95 \%$ CI 55.8-65.3) and CF arms $(57.8 \%, 95 \%$ CI $52.9-62.7 \%)$. However, the cumulative incidence for acute $\geq$ grade 2 GI toxicity was significantly higher (OR 1.6; $p=0.0015)$ in the MH arm $(42 \%, 95 \%$ CI $37.2-$ $46.9 \%)$ compared to control $(31.2 \%, 95 \%$ CI $26.6-35.8 \%)$.
Furthermore, the cumulative incidence of late $\geq$ grade $3 \mathrm{GU}$ toxicity was significantly higher in the MH arm (19 vs $12 \%$; $p=0.021$ ). No statistically significant difference in cumulative $\geq$ grade 3 late gastrointestinal toxicity was found the two study arms ( 2.6 vs $3.3 \%)$.

In contrast to CHHiP [18••, 19, 27] and PROFIT [24•], HYPRO $[20,21,22 \cdot]$ concludes that hypofractionation is not non-inferior to $\mathrm{CF}$, with respect to $\geq$ grade 3 late $\mathrm{GU}$ toxicity.

For similar reasons to the higher toxicity seen in RTOG 0415 [23•], the higher late toxicity in HYPRO [20, 21, 22•] may relate to a higher BED delivered to organs at risk in the hypofractionated arm of HYPRO.

Additionally, compared to CHHiP [18••, 19, 27], HYPRO $[20,21,22 \cdot]$ included a greater proportion of the seminal vesicles in the high-dose volume, which may account for the higher late toxicity. In HYPRO, patients with $>10 \%$ risk of seminal vesicle involvement had their seminal vesicle included in the high-dose volume. Patients with a $10-25 \%$ probability of seminal vesicle involvement received a total dose of 70-72.15 Gy/1.85-2.0 Gy per fraction in the control arm or 54.4-57.76 Gy/3.04-3.4 Gy per fraction in the hypofractionated arm. For patients with a $>25 \%$ risk of seminal vesicle involvement, the seminal vesicles were treated to the full dose of 78 Gy or 64.6 Gy in each respective arm. By contrast, in CHHiP [18••, 19, 27], patients with $>15 \%$ risk of seminal vesicle involvement received $96 \%$ of the prescribed dose to the base of the seminal vesicles, and $80 \%$ of the prescribed dose to the seminal vesicles, within each arm.

The outcome of HYPRO $[20,21,22 \cdot]$ is in keeping with other superiority hypofractionation trials, which did not demonstrate an improvement in efficacy outcomes with hypofractionation. Assuming $\alpha / \beta$ ratio of $1.5 \mathrm{~Gy}$ for $\mathrm{PCa}$, the 2 Gy equivalent dose in the hypofractionated arm of HYPRO is $90.4 \mathrm{~Gy}$. This dose escalation, over the control arm of $78 \mathrm{~Gy} / 39$ fractions, may have been expected to have resulted in a significant improvement in biochemical control. Its failure to do so may relate to the longer duration over which treatment was delivered (6.5 weeks), compared to other hypofractionation schedules.

\section{Extreme Hypofractionation}

Extreme hypofractionation $(\mathrm{EH})$ using stereotactic body radiotherapy (SBRT) refers to the delivery of 6-10 Gy per fraction either daily, on alternate days or weekly, to a total dose of 3550 Gy (Fig. 1). While the linear-quadratic model predicts an improvement in therapeutic ratio with $\mathrm{MH}$, its applicability to EH has been questioned [32], because it does not account for vascular and stromal tissue injury occurring at $\mathrm{EH}$. However, this criticism relates to doses above $10 \mathrm{~Gy}$ per fraction, which are not used in $\mathrm{EH}$ of $\mathrm{PCa}$ radiotherapy [33]. At doses below 10 Gy per 


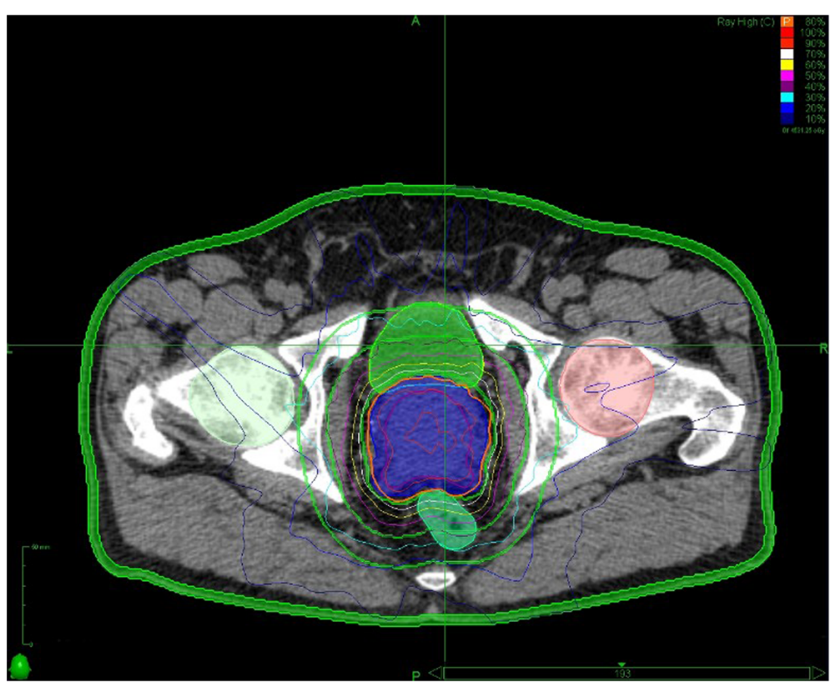

Fig. 1 Treatment plan and dose statistics for prostate SBRT with a noncoplanar technique. (Acknowledgement: Dr Nicholas van As, Kirsty Morrison, Royal Marsden Hospital, UK)

fraction, the linear-quadratic model seems to predict tumour control well, without the need for additional factors [34].

\section{Extreme Hypofractionation for Localized Disease}

Two systematic reviews $[16,17]$ have recommended that EH be only pursued in the setting of a clinical trial, owing to the absence of long-term data. In the last decade, phase I-II data using either IMRT [35-38] or non-coplanar techniques [39-45] have demonstrated toxicity and efficacy outcomes of EH being comparable to CF. Patient-reported quality-of-life outcomes are similar between IMRT, SBRT and LDR brachytherapy at 2 years [46]. SBRT compares favourably with IMRT and LDR brachytherapy in terms physician-reported toxicity outcomes [47].

Outcomes with prostate SBRT have been encouraging for doses between 33.5 and 40 Gy/6.7-8.0 Gy per fraction. Based on a study by Kim [38], doses $>9$ Gy per fraction to a total of $>45 \mathrm{~Gy}$ to the whole prostate are not recommended, owing to higher risk of toxicity. In this dose-escalation SBRT study, patients with low-intermediate-risk disease, received $45 \mathrm{~Gy} / 5$ fractions/9 Gy per fraction, $47.5 \mathrm{~Gy} / 5$ fractions/9.5 Gy per fraction, or $50 \mathrm{~Gy} / 5$ fractions/10 Gy per fraction. After a median follow-up of 24 months, $6.6 \%$ of patients treated with $50 \mathrm{~Gy} / 5$ fractions had $\geq$ grade 3 late rectal toxicity.

Studies treating patients to $33.5-36.25 \mathrm{~Gy} / 5$ fractions/6.77.25 Gy per fraction have reported good early outcomes with acceptable toxicities.

Madsen [35] treated 40 patients to a dose of $33.5 \mathrm{~Gy} / 5$ daily fractions/6.7 Gy per fraction. After a median follow-up of 41 months, biochemical disease-free survival was $90 \%$, with no incidence of $\geq$ grade $3 \mathrm{GI} / \mathrm{GU}$ toxicity. Grade $2 \mathrm{GU} / \mathrm{GI}$ toxicity was reported at 20 and $8 \%$, respectively.
Loblaw [36] treated 84 patients with low-risk disease, with 35 Gy/5 weekly fractions/7 Gy per fraction. After a median follow-up of 55 months, biochemical relapse-free survival was reported at $98 \%$. No acute GU toxicity $\geq$ grade 3 was reported. Late grade $2 \mathrm{GU} / \mathrm{GI}$ toxicity was 7 and $5 \%$, respectively.

Chen [41] treated 100 low-high-risk patients with $36.25 \mathrm{~Gy} / 5$ alternate day fractions/7.25 Gy per fraction. After a median follow-up of 27 months, the biochemical disease-free survival was $99 \%$, with no acute $\geq$ grade $3 \mathrm{GI} /$ GU toxicity. The late $\geq$ grade $3 \mathrm{GU}$ toxicity rate was $1 \%$. Grade 2 acute and chronic GU toxicity was 35 and 31\%, respectively (CTCAE); this is higher than that reported in other studies [40, 44], primarily as it relates to the use of $\alpha$ antagonists in the study. The higher reported toxicity, did not have a significant impact on patients' long-term quality of life [49]. Acute and late GI toxicity was 5 and $1 \%$, respectively.

The above studies measured acute toxicity only once in the first 3 months after SBRT. Studies that have evaluated acute toxicity more than once in the first 3 months after SBRT [42, $50]$ have reported higher toxicity rates.

Bolzicco [42] treated 100 patients with low-high-risk disease, with $35 \mathrm{~Gy} / 5$ daily fractions/7 Gy per fraction. No acute grade 3 toxicity was reported. Acute GI/GU toxicity was 18 and $12 \%$, respectively. Late GI/GU toxicity was 1 and $3 \%$, respectively. The biochemical relapse-free survival was $95 \%$ after a median follow-up of 36 months.

Tree [50] has reported on a series of 51 patients treated with $36.25 \mathrm{~Gy} / 5$ alternate day fractions/7.25 Gy per fraction. Acute grade $2 \mathrm{GU} / \mathrm{GI}$ toxicity was 22 and $14 \%$, respectively, with $4 \%$ of patients having acute grade 3 urinary toxicity.

Long-term outcomes for prostate SBRT have been reported by Meier [45] and King [48].

A multi-institutional study [45] recruited 309 patients, with low-intermediate-risk disease, treating the prostate to $40 \mathrm{~Gy} / 5$ fractions $/ 8$ Gy per fraction and the seminal vesicles to $36.25 \mathrm{~Gy} / 5$ fractions/7.25 Gy per fraction. After a median follow-up of 61 months, $1.6 \%$ of patients reported grade 3 toxicity, all of which were GU toxicities. No grade 4-5 toxicity was reported. The 5-year overall survival and biochemical disease-free survival rates were 95.6 and $97.1 \%$ respectively.

King performed a pooled analysis of 1100 patients, treated with SBRT [48]. Eleven percent of patients had high-risk disease. At a median follow-up of 36 months, the 5-year biochemical disease-free survival for low-, intermediate- and high-risk disease was 95,84 , and $81 \%$, respectively.

While the phase I-II data on prostate SBRT is encouraging, the phase III data is eagerly awaited. There are two phase III studies comparing EH with CF.

The Swedish HYPO trial (ISRCTN45905321) has randomized 592 patients, with intermediate-risk disease to $\mathrm{CF}$ or $42.7 \mathrm{~Gy} / 7$ alternate day fractions/6.1 Gy per fraction. 
The PACE B study (NCT01584258) is ongoing, and randomizes patients to $\mathrm{CF}$ or $36.25 \mathrm{~Gy} / 5$ fractions/7.25 Gy per fraction $/ 5$ days. In view of the recently published $\mathrm{CHHiP}$ study [18••], a hypofractionated protocol amendment has been developed for PACE B, allowing $62 \mathrm{~Gy} / 20$ fractions/3.1 Gy per fraction/4 weeks.

The PATRIOT study is evaluating alternate day (treatment duration 11 days) versus weekly (treatment duration 29 days) stereotactic prostate radiotherapy. This study, delivers $40 \mathrm{~Gy} / 5$ fractions/8 Gy per fraction to the prostate and evaluates bowel quality-of-life parameters. Early results suggest superior quality of life with respect to bowel and urinary function, in the first 3 months following treatment, in the 29-day arm of the study [51].

\section{Extreme Hypofractionation in Oligometastatic Disease}

Oligometastatic $\mathrm{PCa}$, referring to $\leq 3$ isolated sites of metastatic disease, is increasingly being diagnosed, partly because of the development of sophisticated imaging techniques. Historically, the treatment of oligometastatic disease has been identical to that of polymetastatic disease; however, oligometastatic disease may represent a biologically distinct clinical state [52]. Aggressive treatment of oligometastases may help eliminate castrate-resistant clones, delay the development of castrate-resistant disease and thereby potentially improve survival [53].

The data on EH for oligometastatic disease is limited. The two largest studies of SBRT in oligometastatic disease each had 50 patients with predominantly nodal or osseous disease $[54,55]$.

Decaestecker [54] treated metastases to $50 \mathrm{~Gy} / 10$ fractions with 1 month of hormonal therapy or $30 \mathrm{~Gy} / 3$ fractions without hormonal therapy. The median progression-free survival was 19 months, and a 2-year progression-free survival of $35 \%$. Grade 2 toxicity was reported in $6 \%$ of patients.

Schick [55] reported 3-year progression-free survival of $59 \%$. In comparison to the Decaestecker study, the Schick study treated a greater proportion of patients (98 vs 70\%) with longer duration of hormonal therapy (1 year vs 1 month). Additionally, in those patients treated for oligometastic nodal disease, more patients received prophylactic irradiation in the Schick study (61 vs 0\%).

Jereczek-Fossa [56] treated 19 patients with predominantly pelvic nodal oligometastatic disease to a dose of 33-36 Gy/3 fractions; all patients were receiving between 12 and 17 months of hormonal therapy. The 30 -month progressionfree survival was $63.5 \%$.

Casamassima [57] treated 25 patients with nodal oligometastatic disease, to $30 \mathrm{~Gy} / 3$ fractions, without hormonal therapy. The median progression-free survival was 24 months, with no $\geq$ grade 2 toxicity.

Muacevic [58] reported on 40 patients with mainly spinal oligometastatic osseous disease. An actuarial 2-year local tumour control rate, as measured by MRI and PET-CT was reported at $95.5 \%$.

A systematic review of retrospective studies [59] reported on 299 patients with oligometastatic disease treated with radiotherapy, $55 \%$ of whom were treated with extreme hypofractionation. Of the data available for acute toxicity, grade $1-2$ toxicity was reported in $15 \%$ of patients, with grade 3 toxicity in $0.7 \%$.

Pooled analysis of 119 patients, with $\leq 3$ metastases, treated with SBRT has been reported by Ost $[60,61]$. The local control was found to be superior when SBRT delivered a BED $\geq 100$ Gy. No $\geq$ grade 3 toxicities were reported, with $3 \%$ of patients reporting grade 2 toxicity. The 3 -year distant progression-free survival was reported at $31 \%$.

Pooled analysis [60] of a subset of 72 patients with oligorecurrent nodal disease, treated with SBRT at a dose of $5 \mathrm{~Gy}$ per fraction to a BED of at least $80 \mathrm{~Gy}$, has reported a median distant progression-free survival of 21 months, with a progression-free survival of $34 \% / 13 \%$ at $3 / 5$ years, respectively. Late grade 1 and 2 toxicity was reported in 17 and $4 \%$ of patients, respectively.

From these studies, SBRT appears to achieve local control of oligometastatic disease; however, randomized studies are needed to establish its impact on progression-free survival and overall survival.

Several prospective studies are currently underway evaluating SBRT in oligometastatic disease (the phase II CORE study NCT02759783, the SABR-COMET study NCT01446744, the STOMP study NCT01558427, the ORIOLE study NCT02680587).

\section{Conclusions}

Moderate hypofractionation for localized PCa is safe and effective. Based on several large randomized studies, hypofractionation at around 3 Gy per fraction can be considered a standard of care for localized PCa.

There is a growing body of evidence in support of extreme hypofractionation for localized PCa. Comparative data and the data from several randomized studies are awaited. Prostate oligometastases represent another scenario where extreme hypofractionation may have a role but the results of ongoing studies are needed before this is considered the standard of care.

Acknowledgements This work was undertaken in The Royal Marsden NHS Foundation Trust who received a proportion of its funding from the NHS Executive; the views expressed in this publication are those of the authors and not necessarily those of the NHS Executive. This work was supported by The Institute of Cancer Research, the Bob Champion Cancer Trust and Cancer Research UK Section of Radiotherapy [CRUK] grant number C33589/A19727. 
We acknowledge NHS funding to the NIHR Biomedical Research Centre at the Royal Marsden NHS Foundation Trust and Institute of Cancer Research and Cancer Research UK Section of Radiotherapy Programme Grants C33589/A19727, C46/A10588 and C46/A2131.

\section{Compliance with Ethical Standards}

Conflict of Interest Linus C. Benjamin declares that he has no conflict of interest.

Alison Tree has received support through grants from Accuray and Elekta, and has received travel assistance for meetings from Elekta.

David P. Dearnaley declares that he has no conflict of interest.

Human and Animal Rights and Informed Consent This article does not contain any studies with human or animal subjects performed by any of the authors.

Open Access This article is distributed under the terms of the Creative Commons Attribution 4.0 International License (http:// creativecommons.org/licenses/by/4.0/), which permits unrestricted use, distribution, and reproduction in any medium, provided you give appropriate credit to the original author(s) and the source, provide a link to the Creative Commons license, and indicate if changes were made.

\section{References}

Papers of particular interest, published recently, have been highlighted as:

- Of importance

•- Of major importance

1. Jemal A, Bray F, Center MM, Ferlay J, Ward E, Forman D. Global cancer statistics. CA Cancer J Clin. 2011;61(2):69-90.

2. Schröder FH et al. Screening and prostate-cancer mortality in a randomized European study. N Engl J Med. 2009;360(13):1320-8.

3. Withers HR. Biologic basis for altered fractionation schemes. Cancer. 1985;55(S9):2086-95.

4. Thames HD, Withers HR, Peters LJ, Fletcher GH. Changes in early and late radiation responses with altered dose fractionation: implications for dose-survival relationships. Int J Radiat Oncol Biol Phys. 1982;8(2):219-26.

5. Fowler JF. The linear-quadratic formula and progress in fractionated radiotherapy. Br J Radiol. 1989;62(740):679-94.

6. Kuban DA et al. Long-term failure patterns and survival in a randomized dose-escalation trial for prostate cancer. Who dies of disease? Int J Radiat Oncol Biol Phys. 2011;79(5):1310-7.

7. Zietman $\mathrm{AL}$ et al. Randomized trial comparing conventional-dose with high-dose conformal radiation therapy in early-stage adenocarcinoma of the prostate: long-term results from proton radiation oncology group/American college of radiology 95-09. J Clin Oncol. 2010;28(7):1106-11.

8. Dearnaley DP et al. Escalated-dose versus control-dose conformal radiotherapy for prostate cancer: long-term results from the MRC RT01 randomised controlled trial. Lancet Oncol. 2014;15(4):464-73.

9. Al-Mamgani A, van Putten WL, van der Wielen GJ, Levendag PC, Incrocci L. Dose escalation and quality of life in patients with localized prostate cancer treated with radiotherapy: long-term results of the Dutch randomized dose-escalation trial (CKTO 96-10 trial). Int J Radiat Oncol Biol Phys. 2011;79(4):1004-12.
10. Brenner DJ, Hall EJ. Fractionation and protraction for radiotherapy of prostate carcinoma. Int J Radiat Oncol Biol Phys. 1999;43(5): 1095-101.

11. Fowler J, Chappell R, Ritter M. Is $\alpha / \beta$ for prostate tumors really low? Int J Radiat Oncol Biol Phys. 2001;50(4):1021-31.

12. Miralbell R, Roberts SA, Zubizarreta E, Hendry JH. Dosefractionation sensitivity of prostate cancer deduced from radiotherapy outcomes of 5,969 patients in seven international institutional datasets: $\alpha / \beta=1.4(0.9-2.2)$ Gy. Int J Radiat Oncol Biol Phys. 2012;82(1):e17-24.

13. Proust-Lima $C$ et al. Confirmation of a low $\alpha / \beta$ ratio for prostate cancer treated by external beam radiation therapy alone using a post-treatment repeated-measures model for PSA dynamics. Int J Radiat Oncol Biol Phys. 2011;79(1):195-201.

14. Vogelius IR, Bentzen SM. Meta-analysis of the alpha/beta ratio for prostate cancer in the presence of an overall time factor: bad news, good news, or no news? Int J Radiat Oncol Biol Phys. 2013;85(1): 89-94.

15. Tree AC, Alexander EJ, Van As NJ, Dearnaley DP, Khoo V. Biological dose escalation and hypofractionation: what is there to be gained and how will it best be done? Clin Oncol. 2013;25(8): 483-98.

16. Zaorsky NG, Ohri N, Showalter TN, Dicker AP, Den RB. Systematic review of hypofractionated radiation therapy for prostate cancer. Cancer Treat Rev. 2013;39(7):728-36.

17. Koontz BF, Bossi A, Cozzarini C, Wiegel T, D'Amico A. A systematic review of hypofractionation for primary management of prostate cancer. Eur Urol. 2015;68(4):683-91.

18.• Dearnaley D et al. Conventional versus hypofractionated high-dose intensity-modulated radiotherapy for prostate cancer: 5-year outcomes of the randomised, non-inferiority, phase 3 CHHiP trial. Lancet Oncol. 2016;17(8):1047-60. The CHHiP trial is the largest non-inferiority randomized controlled trial to date. CHHiP demonstrated that moderate hypofractionation was noninferior to conventional fractionation and provides the evidence base for recommending $60 \mathrm{~Gy}$ in 20 fractions as the new standard of care for external-beam radiotherapy of localised prostate cancer. The $\alpha / \beta$ for prostate cancer estimated by the CHHiP data is $1.8 \mathrm{~Gy}$.

19. Wilkins A et al. Hypofractionated radiotherapy versus conventionally fractionated radiotherapy for patients with intermediate-risk localised prostate cancer: 2-year patient-reported outcomes of the randomised, non-inferiority, phase 3 CHHiP trial. Lancet Oncol. 2015;16(16):1605-16.

20. Aluwini $\mathrm{S}$ et al. Hypofractionated versus conventionally fractionated radiotherapy for patients with prostate cancer (HYPRO): acute toxicity results from a randomised non-inferiority phase 3 trial. Lancet Oncol. 2015;16(3):274-83.

21. Aluwini S et al. Hypofractionated versus conventionally fractionated radiotherapy for patients with prostate cancer (HYPRO): late toxicity results from a randomised, non-inferiority, phase 3 trial. Lancet Oncol. 2016;17(4):464-74.

22. Incrocci $\mathrm{L}$ et al. Hypofractionated versus conventionally fractionated radiotherapy for patients with localised prostate cancer (HYPRO): final efficacy results from a randomised, multicentre, open-label, phase 3 trial. Lancet Oncol. 2016;17(8):1061-9. The HYPRO study is the largest of the superiority randomized controlled trials. It demonstrated no improvement in efficacy outcomes with hypofractionation. Cumulative acute $\geq$ grade 2 GI toxicity and cumulative late $\geq$ grade $3 \mathrm{GU}$ toxicity was higher in the moderate hypofractionation arm.

23. Lee WR, et al. Randomized phase III noninferiority study comparing two radiotherapy fractionation schedules in patients with low-risk prostate cancer. J Clin Oncol 2016;JCO670448. RTOG 0415 demonstrated that the efficacy of hypofractionation was non-inferior to conventional fractionation in low-risk prostate cancer. Late grade 2-3 
GI/GU toxicity was higher in the hypofractionated arm. The BED to the rectum/bladder was higher in the hypofractionated arm compared to the control arm.

24. Catton $\mathrm{CN}$, et al. A randomized trial of a shorter radiation fractionation schedule for the treatment of localized prostate cancer. $\mathrm{J}$ ClinOncol 2016;34(suppl; abstr 5003). The PROFIT study demonstrates no differences in efficacy and acute toxicity between moderate hypofractionation and conventionally-fractionated radiotherapy in intermediate-risk prostate cancer. Late GI/ GU toxicity was lower in the hypofractionated arm.

25. Lukka $\mathrm{H}$ et al. Randomized trial comparing two fractionation schedules for patients with localized prostate cancer. J Clin Oncol. 2005;23(25):6132-8.

26. Yeoh EE, Botten RJ, Butters J, Di Matteo AC, Holloway RH, Fowler J. Hypofractionated versus conventionally fractionated radiotherapy for prostate carcinoma: final results of phase III randomized trial. Int J Radiat Oncol Biol Phys. 2011;81(5):1271-8.

27. Dearnaley $\mathrm{D}$ et al. Conventional versus hypofractionated high-dose intensity-modulated radiotherapy for prostate cancer: preliminary safety results from the CHHiP randomised controlled trial. Lancet Oncol. 2012;13(1):43-54

28. Kuban DA et al. Preliminary report of a randomized dose escalation trial for prostate cancer using hypofractionation. Int J Radiat Oncol Biol Phys. 2010;78(3):S58-9.

29. Hoffman KE et al. Risk of late toxicity in men receiving doseescalated hypofractionated intensity modulated prostate radiation therapy: results from a randomized trial. Int $\mathrm{J}$ Radiat Oncol Biol Phys. 2014;88(5):1074-84.

30. Arcangeli S et al. Updated results and patterns of failure in a randomized hypofractionation trial for high-risk prostate cancer. Int $\mathbf{J}$ Radiat Oncol Biol Phys. 2012;84(5):1172-8.

31. Pollack A, et al. Randomized trial of hypofractionated external-beam radiotherapy for prostate cancer. J Clin Oncol 2013;JCO-2013.

32. Kirkpatrick JP, Meyer JJ, Marks LB. The linear-quadratic model is inappropriate to model high dose per fraction effects in radiosurgery. Semin Radiat Oncol. 2008;18:240-3.

33. Brenner DJ. The linear-quadratic model is an appropriate methodology for determining isoeffective doses at large doses per fraction. Semin Radiat Oncol. 2008;18:234-9.

34. Brown JM, Carlson DJ, Brenner DJ. The tumor radiobiology of SRS and SBRT: are more than the 5 Rs involved? Int J Radiat Oncol Biol Phys. 2014;88(2):254-62.

35. Madsen BL, Hsi RA, Pham HT, Fowler JF, Esagui L, Corman J. Stereotactic hypofractionated accurate radiotherapy of the prostate (SHARP), $33.5 \mathrm{~Gy}$ in five fractions for localized disease: first clinical trial results. Int J Radiat Oncol Biol Phys. 2007;67(4):1099-105.

36. Loblaw A et al. Prostate stereotactic ablative body radiotherapy using a standard linear accelerator: toxicity, biochemical, and pathological outcomes. Radiother Oncol. 2013;107(2):153-8.

37. Aluwini S, van Rooij P, Hoogeman M, Kirkels W, KolkmanDeurloo I-K, Bangma C. Stereotactic body radiotherapy with a focal boost to the MRI-visible tumor as monotherapy for low-and intermediate-risk prostate cancer: early results. Radiat Oncol. 2013;8(1):1.

38. Kim DN et al. Predictors of rectal tolerance observed in a doseescalated phase 1-2 trial of stereotactic body radiation therapy for prostate cancer. Int J Radiat Oncol Biol Phys. 2014;89(3):509-17.

39. Fuller DB, Naitoh J, Mardirossian G. Virtual HDR CyberKnife SBRT for localized prostatic carcinoma: 5-year disease-free survival and toxicity observations. Front Oncol. 2014:4:321.

40. King CR, Brooks JD, Gill H, Presti JC. Long-term outcomes from a prospective trial of stereotactic body radiotherapy for low-risk prostate cancer. Int J Radiat Oncol Biol Phys. 2012;82(2):877-82.
41. Chen LN et al. Stereotactic body radiation therapy (SBRT) for clinically localized prostate cancer: the Georgetown University experience. Radiat Oncol. 2013;8(1):1.

42. Bolzicco G, Favretto MS, Satariano N, Scremin E, Tambone C, Tasca A. A single-center study of 100 consecutive patients with localized prostate cancer treated with stereotactic body radiotherapy. BMC Urol. 2013;13(1):1.

43. Meier $\mathrm{R}$ et al. Stereotactic body radiation therapy for intermediaterisk prostate cancer: five-year outcomes from a multi-institutional study. Int J Radiat Oncol Biol Phys. 2015;93:S199.

44. Katz AJ, Kang J. Quality of life and toxicity after SBRT for organconfined prostate cancer, a 7-year study. Front Oncol. 2014;4:301.

45. Meier R et al. Five-year outcomes from a multicenter trial of stereotactic body radiation therapy for low-and intermediate-risk prostate cancer. Int J Radiat Oncol Biol Phys. 2016;96(2S):S33.

46. Evans JR et al. Patient-reported quality of life after stereotactic body radiotherapy (SBRT), intensity modulated radiotherapy (IMRT), and brachytherapy. Radiother Oncol. 2015;116(2):179-84.

47. Meier R. Dose-escalated robotic SBRT for Stage I-II prostate cancer. Front Oncol 2015;5.

48. King CR et al. Stereotactic body radiotherapy for localized prostate cancer: pooled analysis from a multi-institutional consortium of prospective phase II trials. Radiother Oncol. 2013;109(2):217-21.

49. Arscott WT et al. Obstructive voiding symptoms following stereotactic body radiation therapy for prostate cancer. Radiat Oncol Lond Engl. 2014;9:163.

50. Tree AC et al. Prostate stereotactic body radiotherapy-first UK experience. Clin Oncol. 2014;26(12):757-61.

51. Quon $\mathrm{HC}$ et al. PATRIOT Trial: randomized phase II study of prostate stereotactic body radiotherapy comparing 11 versus 29 days overall treatment time. J Clin Oncol. 2015;33 suppl 7:abstr 6.

52. Lussier YA et al. Oligo-and polymetastatic progression in lung metastasis (es) patients is associated with specific microRNAs. PLoS One. 2012;7(12):e50141.

53. Furuya $\mathrm{Y}$, Akakura $\mathrm{K}$, Akimoto S, Inomiya H, Ito H. Pattern of progression and survival in hormonally treated metastatic prostate cancer. Int J Urol. 1999;6(5):240-4.

54. Decaestecker $\mathrm{K}$ et al. Repeated stereotactic body radiotherapy for oligometastatic prostate cancer recurrence. Radiat Oncol. 2014;9(1):1.

55. Schick $U$ et al. Androgen deprivation and high-dose radiotherapy for oligometastatic prostate cancer patients with less than five regional and/or distant metastases. Acta Oncol. 2013;52(8):1622-8.

56. Jereczek-Fossa BA et al. Robotic image-guided stereotactic radiotherapy, for isolated recurrent primary, lymph node or metastatic prostate cancer. Int J Radiat Oncol Biol Phys. 2012;82(2):889-97.

57. Casamassima F et al. Efficacy of eradicative radiotherapy for limited nodal metastases detected with choline PET scan in prostate cancer patients. Tumori. 2011;97(1):49.

58. Muacevic A, Kufeld M, Rist C, Wowra B, Stief C, Staehler M. Safety and feasibility of image-guided robotic radiosurgery for patients with limited bone metastases of prostate cancer. Urol Oncol. 2013;31(4):455-60.

59. Ost $\mathrm{P}$ et al. Metastasis-directed therapy of regional and distant recurrences after curative treatment of prostate cancer: a systematic review of the literature. Eur Urol. 2015;67(5):852-63.

60. Ost $\mathrm{P}$ et al. Pattern of progression after stereotactic body radiotherapy for oligometastatic prostate cancer nodal recurrences. Clin Oncol (R Coll Radiol). 2016;28(9):e115-120.

61. Ost $\mathrm{P}$ et al. Progression-free survival following stereotactic body radiotherapy for oligometastatic prostate cancer treatment-naive recurrence: a multi-institutional analysis. Eur Urol. 2016;69(1):9-12. 